\title{
Pour une rhétorique sémiotique de l'image en sciences biologiques
}

Developing a Semiotic Rhetoric of Images in the Biosciences

\section{Maria Giulia Dondero}

\section{(2) OpenEdition \\ Journals}

Electronic version

URL: http://journals.openedition.org/aad/2169

DOI: 10.4000/aad.2169

ISSN: 1565-8961

Publisher

Université de Tel-Aviv

\section{Electronic reference}

Maria Giulia Dondero, "Pour une rhétorique sémiotique de l'image en sciences biologiques », Argumentation et Analyse du Discours [Online], 16 | 2016, Online since 09 April 2016, connection on 23 September 2019. URL : http://journals.openedition.org/aad/2169; DOI : 10.4000/aad.2169

This text was automatically generated on 23 September 2019.

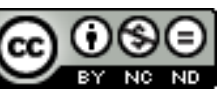

Argumentation \& analyse du discours est mis à disposition selon les termes de la licence Creative Commons Attribution - Pas d'Utilisation Commerciale - Pas de Modification 4.0 International. 


\title{
Pour une rhétorique sémiotique de l'image en sciences biologiques
}

Developing a Semiotic Rhetoric of Images in the Biosciences

\author{
Maria Giulia Dondero
}

\section{AUTHOR'S NOTE}

Je remercie chaleureusement Marion Colas-Blaise pour la relecture de ce texte et pour les précieux conseils prodigués

\section{Introduction}

1 Cet article se propose d'analyser la rhétorique des images du vivant en sciences biologiques dans deux genres discursifs distincts: l'article de recherche et le manuel de vulgarisation. Dans un premier temps, nous illustrerons le cadre épistémologique de notre travail, emprunté notamment à la sociologie des sciences de Bruno Latour et à la sémiotique de l'image scientifique élaborée par Françoise Bastide. Ce cadre épistémologique s'associe à l'orientation théorique et méthodologique de la sémiotique du discours post-greimassienne, ainsi qu'à la théorie rhétorique de Jean-François Bordron.

2 Dans un second temps, nous étudierons les opérations rhétoriques (addition, suppression, sélection, superposition, etc.), mises en scène par notre corpus d'images. Plus précisément, nous analyserons un article de recherche sur le système immunitaire et la colonisation du thymus ${ }^{1}$, publié dans la revue Immunity par des biologistes du Laboratoire "Développement du Système Immunitaire » à l'Institut Universitaire d'Hématologie de l'Hôpital Saint-Louis à Paris². Cette recherche n'a pas encore fait l'objet d'un quelconque type de vulgarisation: elle ne dispose, comme diffusion de résultats et instrument de publication, que de quelques articles de recherche publiés dans des revues spécialisées telles que Immunity ou Nature Immunology. Nous 
analyserons les images et notamment le dispositif du "cadre dans le cadre " pour comprendre la manière dont ces images interrogent et déploient l'objet de recherche, et le transformentenobjet scientifique proposé à la communauté scientifique en vue de la discussion et du partage ${ }^{3}$. Nous prendrons ensuite en considération, plus brièvement, le genre discursif du manuel qui ne traite pas des questions pointues de la colonisation du thymus - lesquelles, on l'a dit, ne sont pas encore suffisamment stabilisées dans la communauté scientifique pour pouvoir entrer dans l'univers de la vulgarisation -, mais de questions qui ont préparé le terrain pour l'article de recherche.

\section{Prolégomènes épistémologiques}

3 Comme indiqué plus haut, les travaux qui ont nourri notre analyse des images dans les différents genres du discours scientifique proviennent de différentes disciplines ${ }^{4}$ et notamment de la sociologie/anthropologie des sciences de Bruno Latour et de la sémiotique du discours scientifique de Françoise Bastide ${ }^{5}$. Ces travaux s'ancrent en partie dans la sémiotique narrative et énonciative d'Algirdas Julien Greimas et de l'École de Paris, notamment en ce qui concerne la conception de la référence. Nous aborderons ci-dessous quatre prises de position épistémologiques que nous partageons avec la théorie de Latour et avec la sémiotique du discours greimassienne et postgreimassienne et qui préparent le terrain pour la partie analytique.

\subsection{La référence de l'image}

4 Tout d'abord la question de la référence de l'image. Comment peut-on faire émerger la référence dans/par des visualisations? Selon Latour, la pratique de la recherche scientifique peut se concevoir comme un cheminement à travers des chaînes d'observateurs ${ }^{6}$ et des médiateurs ; la référence n'est donc pas conçue à « l'origine » de l'image, à l'instar d'une source stable en attente d'être captée, mais comme quelque chose qui circule entre les médiateurs qui essaient d'en explorer le fonctionnement. L'image est un de ces médiateurs au travers desquels la référence se déploie, se transforme, se précise, en réponse aux questionnements et aux hypothèses formulées par les scientifiques. Comme l'affirment Latour et Hermant dans Paris Ville Invisible :

Certes, le phénomène n'apparaît jamais sur l'image, mais il devient pourtant visible dans ce qui se transforme, se transporte, se déforme d'une image à l'autre, d'un point de vue, d'une perspective à l'autre. Il faut qu'une trace les relie, permette d'aller et de venir, de circuler le long de cette voie-là [...], transversale, latérale (Latour et Hermant $1998: 53$, nous soulignons).

5 Si on suit cette conception de la référence, il est clair qu'aucune référence ne se manifestera jamais dans une seule image. Comme l'affirment Latour et Hermant, "l'idolâtrie et l'iconolâtrie consistent à croire en une seule image comme si c'était la bonne » (53). La recherche expérimentale serait donc à entendre comme un ensemble de mouvements, de déplacements, d'alignements des traces, suivi de mouvements de réassemblage de ces traces : c'est le réassemblage des traces qui constitue ce qu'on peut in fine appeler « la référence » ou bien « un objet de recherche constitué », un objet qui, pendant les déplacements, est en revanche en train d'être exploré, testé, manipulé.

6 Dans ses articles, Bastide (2001) affirme que la fidélité de la représentation des procès du monde naturel dépend de la syntaxe des dispositifs traducteurs et médiateurs 
utilisés. Dans cette chaîne de médiateurs et de traces, aucun des points de vue adoptés, aucune image ne donne une vision de la totalité de l'objet de recherche mais plutôt du point de vue précédent qu'elle est en train de transformer.

7 Pourtant, selon Latour $(1999,2012)$, ces chaînes de médiateurs finissent par construire une forme, voire une sorte de totalité, à savoir un objet scientifique constitué, stabilisé par différents tests et expériences. Nous pourrions avancer l'idée que les opérations d'expérimentation amènent à la constitution d'un objet au sens où les opérations offrent aux chercheurs des réponses qui prennent forme, qui se stabilisent en totalités. Mais cette forme apparait exclusivement suite à un mouvement de stabilisation des expérimentations. Elle peut être entendue comme chaîne d'images «qui se tient » et qui constitue une épreuve en soi, car elle assure le contrôle mutuel d'une image vis-àvis de l'autre.

8 Une fois quittée l'expérimentation, commence le travail de présentation des résultats, qui sont normalement discutés dans des rapports de laboratoire et qui trouvent ensuite une première stabilisation dans l'article de recherche. Une seconde forme de stabilisation, voire de consolidation - éventuelle, car elle dépend d'un processus d'acceptation, plus ou moins long, de la part de la communauté des collègues - verra le jour ensuite dans le domaine de la vulgarisation. Comme nous le verrons plus loin, les genres de la vulgarisation (plus ou moins savante) sont caractérisés par des formes discursives qui ne laissent plus beaucoup de place aux chaînes d'images et à l'explication des processus de laboratoire. Dans la vulgarisation, la chaîne d'images est souvent stabilisée en une seule image qui «résume » ou prend la place des images utilisées dans l'article de recherche. Or, cette image, qui se présente comme définissant l'objet de recherche, est souvent le résultat de la stabilisation de la chaîne d'images.

9 Il est clair que, suivant cette conception du travail scientifique, le référent n'est jamais déjà constitué en amont de la recherche, mais est en revanche concevable comme le résultat de ce qui se transforme suite aux opérations des chercheurs et de leurs instruments - et notamment des procédures de l'inter-instrumentalité, qui permet une vérification mutuelle des expérimentations. Ce qu'on appelle normalement le référent de l'image scientifique peut être entendu comme une référencerésultant de trois sortes de manipulations : la manipulation des phénomènes physiques, les négociations entre chercheurs et l'inter-instrumentalité 7 . La référence en science est ainsi définissable comme ce qui fait face aux expériences du scientifiqueet résiste à de mauvaises manipulations/descriptions.

10 Si cette première prise de position porte sur le passage entre le travail expérimental du scientifique en laboratoire et la présentation des résultats, la deuxième concerne, par contre, le discours du scientifique, voire la présentation des pratiques de recherche par la voie des images.

\subsection{L'intertextualité}

11 La deuxième prise de position concerne la textualisation de la référence dans les publications scientifiques, qui peut s'entendre non seulement comme un résultat des manipulations ayant cours au laboratoire, mais également comme un produit de l'intertextualité, à savoir du dialogue avec des textes scientifiques publiés précédemment ainsi qu'avec des textes possibles qui pourraient critiquer le texte en préparation. Pour Bastide, en effet, le scientifique est un opérateur textuel et 
intertextuel qui accomplit un travail de composition et de décomposition de la réalité physique ainsi que du substrat des débats ayant lieu dans la littérature spécialisée. Cette intertextualité est irriguée de conflits. Dans ses analyses, Bastide (1985) montre en effet que tout texte écrit par un scientifique construit des controverses internes implicites: les anti-programmes et les anti-sujets présents au sein des articles scientifiques - ces anti-programmes pouvant être identifiés à la fois dans des matériaux, dans des durées alternatives des processus d'expérimentation, ou bien dans des coupes temporelles alternatives à celles choisies par des chercheurs concurrents réfléchissent une situation conflictuelle externe au discours, et qu'on appelle communément controverse.

12 Bastide (1985) affirme à ce propos qu'un article scientifique, avant d'être contesté, constitue un tout unique. Lorsque, par contre, des collègues concurrents mettent en danger l'identité d'un matériau identifié par l'article à travers une photo par exemple, ils détruisent l'unité du dispositif, l'article se scindant dans ses composantes expérimentales. On pourrait affirmer que la controverse met en échec la totalité de l'expérimentation et de sa publication : la contestation fait en sorte que chaque passage de la démonstration du scientifique subit une bifurcation, ces bifurcations signalant les points ou nœuds où peuvent s'introduire, par exemple, une erreur d'interprétation ou bien une fraude. Nous nous apercevons que, dans ce cas également, la composition/ décomposition d'une totalité est au cœur de la production et de la réception du texte scientifique.

\subsection{Cadrage, délimitation et focalisation}

13 Une troisième question abordée par Bastide, concernant plus précisément la rhétorique des images scientifiques, concerne le fait que la production de chaque image en laboratoire est toujours le résultat d'un cadrage, voire d'une action de délimitation et de focalisation. Ce qui est intéressant à ce sujet est que cette action de sélection ne concerne pas exclusivement un découpage spatial mais aussi un découpage temporel. La fréquence établie pour des observations de transformations est un exemple de segmentation, voire de cadrage, plus ou moins arbitraire, d'une unité de temps scientifiquement pertinente, sélectionnée dans le continuum du déroulement de l'expérience.

14 À ce propos, dans l'article «Le foie lavé, approche sémiotique d'un texte de sciences expérimentales ", publié en 1979, Bastide décrit une expérimentation de Claude Bernard sur le précurseur du sucre. Devant statuer entre sa possible localisation dans le sang ou dans le foie, Bernard décide de construire un temps-zéro de l'expérience par le lavage d'une zone du foie : il construit ainsi un commencement. Bernard choisit, certes, une section précise du foie à analyser mais aussi un point $\mathrm{T}$ zéro à partir duquel observer les transformations - en déterminant également un temps d'attente pour que les transformations puissent apparaître.

15 Cette question est importante car l'on pense d'habitude qu'une image est la trace des phénomènes à partir d'un référentiel spatial. Il faut concevoir en revanche que le référentiel, entendu en tant que "théâtre de l'apparition " (Bordron 2009), voire en tant que lieu de manifestation de la réponse aux expérimentations, n'est pas exclusivement une affaire d'espace mais également de temps, voire d'aspectualisation de l'observation. Nous verrons par la suite que les différents dispositifs du « cadre dans 
le cadre ", contenus dans chaque image publiée, sont toujours orientés vers le passé ou vers le futur de l'expérimentation (et de la chaîne) : au sein de la chaîne, les images ne sont jamais juxtaposées l'une après l'autre en tant qu'éléments séparés mais bien liées par les différents dispositifs de cadrage, plus ou moins rétensifs ou protensifs.

\subsection{Opérations du voir et du savoir}

16 Un quatrième point développé par Bastide (1979) concerne l'homologation entre les opérations du voir et les opérations du savoir. Pour bien voir, il faut délimiter, focaliser, en un mot encadrer, ce qui est l'action fondamentale permise par l'image. À chaque étape, à chaque image de l'expérience, il faut sélectionner ce qui est pertinent pour la composition de l'objet de recherche en cours de transformation et en même temps en cours d'expérimentation. La difficulté est que l'objet visé et ses bords changent en même temps que l'exploration du scientifique, car l'objet réagit à l'expérimentation.

Délimiter et focaliser ne sont pas les seules actions permises par l'image; il ne s'agit donc pas exclusivement d'établir une relation entre le voir, plus ou moins bien, et la modalisation du savoir, plus ou moins certain; il faut aussi considérer que la visualisation met en œuvre d'autres opérations liées à la connaissance telles qu'ajouter/écarter, inclure/exclure, superposer/trier. Ces opérations visent à faire apparaître ce que nous avons appelé une totalité, voire une chaine d'images «qui se tient »- où chaque image est nécessaire pour donner des réponses (partielles) aux problèmes qui ont déclenché la recherche.

\section{La rhétorique méréologique}

Toutes les opérations que nous avons rapidement évoquées (inclure, exclure, ajouter, écarter, séparer) sont au centre de la théorie rhétorique de Jean-François Bordron qui est développée en une théorie de la méréologie, des rapports entre totalité et parties ${ }^{8}$.

Bordron propose d'entendre la notion de rhétorique comme un ensemble d'opérations méréologiques. Dans l'un de ses articles consacrés à la rhétorique visuelle, « Rhétorique et économie des images", il part du tableau esquissé par le Groupe $\mu$ dans l'ouvrage Traité du signe visuel. Pour une rhétorique de l'image (Figure 1): 


\begin{tabular}{|c|c|c|c|c|}
\hline Opérations $\begin{array}{c}\text { Famille de } \\
\text { transfor- } \\
\text { mation }\end{array}$ & Géométriques & Analytiques & Optiques & Cinétiques \\
\hline $\begin{array}{l}\text { Adjonction } \\
\qquad(+)\end{array}$ & 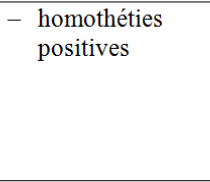 & $\begin{array}{l}\text { - filtrages positifs } \\
\text { - indifférenciations } \\
\text { - continuisations }\end{array}$ & $\begin{array}{l}\text { - } \text { accentuations de } \\
\text { contrastes } \\
\text { - } \text { dilatations de } \\
\text { profondeur } \\
\text { - élargissements du } \\
\text { champ de netteté }\end{array}$ & - \\
\hline $\begin{array}{c}\text { Suppression } \\
(-)\end{array}$ & $\begin{array}{l}\text { - homothéties } \\
\text { négatives }\end{array}$ & $\begin{array}{l}\text { - filtrages négatifs } \\
\text { - différenciations } \\
\text { - discrétisations }\end{array}$ & 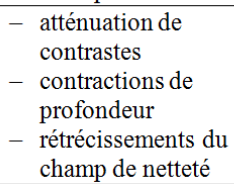 & - \\
\hline $\begin{array}{l}\text { Substitution } \\
( \pm)\end{array}$ & $\begin{array}{l}- \text { projections } \\
- \text { transformations } \\
\text { topologiques }\end{array}$ & $\begin{array}{l}- \text { filtrages } \\
\text { substitutifs }\end{array}$ & $\begin{array}{l}\text { - déplacements du } \\
\text { contraste }\end{array}$ & - \\
\hline $\begin{array}{l}\text { Permutation } \\
(\sim)\end{array}$ & $\begin{array}{l}- \text { translations } \\
\text { - rotations } \\
\text { - déplacements } \\
\text { - congruences }\end{array}$ & & $\begin{array}{l}\text { - inversions } \\
\text { (négatif) }\end{array}$ & $\begin{array}{l}\text { - intégrations } \\
\text { - anamorphoses }\end{array}$ \\
\hline
\end{tabular}

Figure 1. Système de transformations

Source : Groupe H. 1992. Traité du signe visuel. Pour une rhétorique de l'image (Paris : Seuil), p. 157.

En poursuivant sur la voie tracée par la rhétorique du Groupe $\mu$, Bordron affirme :

Il nous semble que la terminologie même utilisée par ces auteurs invite à considérer les opérations rhétoriques [la suppression, l'adjonction, la permutation, la substitution] comme des opérations méréologiques. Il s'agit toujours, plus évidemment chez Perelman, de lier et de délier, de séparer et de conjoindre, de rapprocher et de distancier, de telle sorte que la rhétorique se présente plus comme une gestuelle opérant sur des totalités et sur des parties que comme une logique procédant par démonstration (Bordron $2010: 28$, nous soulignons).

21 Le domaine de la rhétorique est entendu ici comme une méréologie, qui a pour projet « de reconnaître des actes (séparer, fusionner, lier, unifier, partager) portant sur des genres de relations (partie/tout, liaison/diversité, unité/multiplicité, dépendances entre instances) » (ibid. : 39). La rhétorique est ainsi définie comme une gestuelle opérant sur un flux d'expériences - elle "suppose en son fond l'idée d'un objet doué d'une dynamique " (ibid. : 28) -, dont émergeront des formes composées de totalités et de parties.

Ces opérations de manipulation (gestuelle) sont accomplies sur l'objet de recherche qui est encore virtuel, lorsque l'exploration est à ses débuts. La gestuelle rhétorique dont parle Bordron est comparable aux manipulations évoquées par Bastide, dans ses articles sur le faire du scientifique et sur les actions opérées par l'image, cette dernière étant le témoignage d'un théâtre de gestes qui se font, se défont et se refont pour produire un ensemble de compositions multiples de l'objet qui fonctionnent comme des tests de l'objet. Ces compositions multiples d'expériences, qui sont des expérimentations de l'élasticité de l'objet de recherche et de ses limites, se stabiliseront ensuite en une forme unique, voire dans une totalité acceptée et stabilisée, qui le définira dans le cadre de la vulgarisation.

Le travail du scientifique serait donc comparable à une suite d'opérations telles que l'addition, la soustraction, la suppression, la division... Nous allons tout de suite explorer quelques cas de figure en sciences biologiques pour comprendre davantage ces opérations rhétoriques - qui découlent toutes d'une première opération fondamentale qui est celle du cadrage. 


\section{Rhétorique de l'expérimentation en biologie moléculaire}

Venons-en à l'article de biologie immunitaire, publié dans l'une des plus réputées revues d'immunologie, Immunity, en 2006. L'article examiné, qui a pour titre " Dynamics of Thymus-Colonizing Cells during Human Development ", est publié dans la section «Research Articles» et présente une grande variété d'images concernant différentes populations de cellules immunitaires censées coloniser le thymus avant et après la naissance du fœtus.

Le point de la recherche en très bref : toutes les cellules du système immunitaire se développent dans la moelle osseuse sauf les lymphocytes $\mathrm{T}$, qui se développent dans le thymus. Le thymus est colonisé par des cellules produites dans la moelle osseuse par voie sanguine : il s'agit de comprendre quelle est la cellule qui rentre dans le thymus. Une cellule souche, indifférenciée, rejoint-elle le thymus par un parcours aléatoire, ou bien les cellules colonisant le thymus sont-elles prédéterminées à y aller? On sait que la différenciation cellulaire commence dès le stade de la moelle osseuse, mais comment repérer les cellules qui sont censée être préprogrammées, voire dédiées sélectivement à la colonisation de l'organe du thymus?

Nous avons déjà affirmé qu'aucune des images présentes dans l'article ne correspond à aucun objet singulier, à aucune totalité, que ce soit une cellule, une protéine, l'organe du thymus, ou autre chose. L'objet de recherche correspond en effet à une chaîne d'expériences, souvent trop longue pour être suivie en entier.

Pour la constitution de notre corpus, l'hypothèse que nous faisons afin de limiter la chaîne pertinente à étudier est que la totalité de l'objet en tant qu'objet explorable et connaissable est garantie par l'ensemble des images qui sont au cœur de l'article. On prend donc l'article comme totalité englobante et non pas toutes les expériences sur ce phénomène dans leur ensemble. Les images sélectionnées pour la publication sont par conséquent déjà le résultat d'un cadre dans le cadre, ou plutôt d'un arrêt sur image à l'intérieur de la dynamique d'un flux, voire d'un processus expérimental très long.

B Au début de l'article étudié, l'objet de recherche est encore virtuel et il se constitue discursivement au fur et à mesure des manipulations expérimentales des scientifiques. Nous verrons qu'à l'intérieur de l'article, notre objet de recherche va être découpé, manipulé, rendu élastique. Les visualisations nous montrent les transformations du processus lui-même ou, plus précisément, la manière de le découper, spatialement et temporellement, et de l'analyser. Comme nous l'avons évoqué plus haut, l'image se caractérise premièrement comme lieu d'encadrement, de sélection, de découpage, voire d'isolement des parties et de focalisation de l'attention de l'observateur. Ce fonctionnement est en harmonie avec une des opérations les plus fondamentales en biologie qui est celle d'arriver, via des modèles, à isoler des problèmes, à repérer des bords à l'intérieur du flux de la vie en perpétuelle transformation.

Si l'ensemble d'images publiées dans l'article constitue déjà un "cadre dans le cadre " dans sa globalité par rapport au flux expérientiel du phénomène à étudier, la série d'images de la figure 2 qu'on trouve à la page 226 de l'article examiné, met en revanche en scène un premier type decadre dans le cadre interne au cadrage global. On est face 
ici à différents types de cadrage visant la recherche de la cellule censée coloniser le thymus (Figure 2).
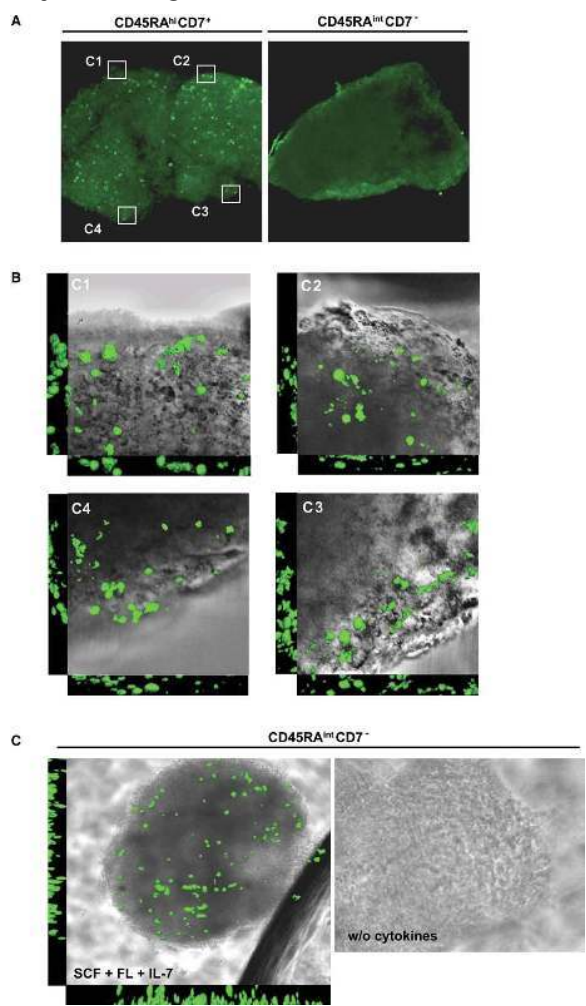

Figure 2. «Ex Vivo Thymus Colonization Assay » (Haddad et alii, 2006 : 226), détail

Dans les deux premières images disposées en haut (groupe A), nous visualisons quatre petits rectangles situés aux bords de la forme colorée en vert; ils nous permettent de localiser les zones qui seront focalisées par la suite, sur lesquelles nous sommes censés centrer l'attention pour poursuivre l'exploration, voire l'approfondir.

31 Les quatre images du groupe $B$ que l'on trouve en dessous des deux premières $(C 1, C 2$, C3, C4) sont en effet engendrées par les deux d'en haut par focalisation et zoom. Ces quatre images nous permettent d'obtenir non seulement une meilleure vision/ focalisation en termes de définition de la qualité de vision (la vision microscopique est plus avancée), mais elles permettent une ouverture sur une nouvelle scène, sur un processus dans le processus.

32 Ce fonctionnement pourrait être rapproché de celui permis par le dispositif de la fenêtre (ces cadres sont d'ailleurs appelés fenêtres par les chercheurs). Si dans le domaine des images artistiques, la représentation de la fenêtre renvoie à une ouverture vers l'altérité, voire à une ouverture de l'intérieur vers l'extérieur ${ }^{10}$, dans l'image en biologie, la fenêtre fonctionne comme une ouverture vers l'intérieur du processus étudié ainsi que, sur le plan temporel, comme l'indicateur d'un processus de projection en avant de la recherche, d'un acte de devancement qui trouvera sa suite dans d'autres images. Ces focalisations fonctionnent ainsi comme des dispositifs de multiplication de nouvelles scènes qui peuvent à leur tour offrir l'accès à des scènes ultérieures, par l'ouverture d'autres fenêtres. Ces images creusent à l'intérieur de l'objet à analyser et en même temps ouvrent sur de nouveaux terrains d'exploration, en relançant la chaîne. En parallèle, l'opération de focalisation et de valorisation d'une partie du processus d'exploration engendre en même temps la soustraction des zones sélectionnées par les 
fenêtres par rapport au fond vert, le fond étant le lieu de l'image où il n'y a pas d'acteurs de la transformation. Dans C1, C2, C3 et C4 (groupe B), nous avons, d'un côté, gagné en profondeur de la vision par rapport aux images du groupe $\mathrm{A}$, en intensité pourrait-on dire, mais nous avons, de l'autre, perdu l'étendue de la vision que nous avions obtenue via les deux premières images du groupe A. Les images du groupe B ont provisoirement perdu la totalité qui était constituée, dans les images d'en haut, du tissu coloré en vert.

Nous découvrons avec $\mathrm{C} 1, \mathrm{C} 2, \mathrm{C} 3$ et $\mathrm{C} 4$ un autre type d'encadrement, qui fonctionne de manière différente de l'encadrement "à fenêtre ": il s'agit d'un encadrement par superposition. Nous sommes ainsi face à deux fonds superposés, l'un noir et l'autre gris. Les cellules des images $\mathrm{C} 1, \mathrm{C} 2, \mathrm{C} 3$ et $\mathrm{C} 4$ se manifestent à travers une action de superposition du fond (gris) sur un autre fond (noir). C1, C2, C3 et C4 mettent en valeur (en gris et en vert) une population de cellules qui, après excitation, permettra à certaines protéines de se fixer sur l'organe du thymus. Comment expliquer le fonctionnement des fonds noirs sur lesquels émergent des fonds gris clair, sur lesquels émergent à leur tour les concrétions en vert obtenues par fluorescence ? C'est ce fond noir en partie inaccessible qui permet l'émergence du fond gris qui fait face à l'observateur et qui s'affiche comme quelque chose possédant un degré de stabilisation plus marqué - car en saillie - que ce qui se passe dans le fond noir.

Il faut également remarquer que chaque groupe d'images (A et B) expose un double cadre: dans les deux premières images $(\mathrm{A})$, nous sommes face à une totalité produite par le tissu vert, qui est en attente de focalisation des quatre parties sélectionnées par les carrés - ce qui amène à une opération de soustraction de la totalité. Les images $\mathrm{C} 1$, C2, C3 et C4 sont précisément le produit de l'extraction d'une totalité provisoire. Elles ont également besoin d'un cadre comme base fonctionnant, d'un côté, comme mémoire de la totalité de laquelle elles ont été extraites et, de l'autre, comme présentoir affichant ce que les nouvelles observations par focalisation permettent de voir.

En ce qui concerne ce que nous avons appelé le découpage temporel au début de notre travail, les images d'en haut (groupe A) hébergent des cadres qu'on pourrait appeler protensifs, c'est-à-dire des cadres allant vers l'avenir de l'expérimentation (groupe B), tandis que les secondes (groupe B) recherchent en revanche un ancrage, via le fond noir, dans le groupe A : elles se superposent sur des fonds qui leur servent de mémoire. Ces derniers types de cadrages peuvent être appelés rétensifs.

37 Cette série d'images est en effet exemplaire du fonctionnement que nous avons esquissé plus haut : le phénomène n'apparaît jamais sur l'image, mais il devient visible dans ce qui se transforme, se transporte, se déforme, d'une image à l'autre, d'un point de vue, d'une perspective à l'autre. Nous pourrions affirmer que les images du groupe $\mathrm{A}$ sont en attente, elles préfigurent des images à venir, un mouvement en avant. En revanche, les images du groupe B sont une sorte de déploiement/approfondissement des premières, dont elles gardent soigneusement les traces : $\mathrm{C} 1, \mathrm{C} 2, \mathrm{C} 3$ et $\mathrm{C} 4$ gardent en mémoire la trace d'une sorte de micro-totalité qui les a engendrées. Elles produisent un mouvement en avant et en arrière. Ce qui est significatif dans cet exemple est que les actes d'encadrement peuvent signifier plusieurs sortes d'opérations, plus ou moins protensives ou rétensives.

38 Dès ces premiers exemples, la pertinence de la rhétorique formulée par Jean-François Bordron devient évidente. Cette rhétorique est entendue comme gestuelle, voire comme un ensemble d'opérations de manipulation constituant des morphologies 
discursives à partir d'un flux expérientiel. Autrement dit, cette gestuelle - qui est une action de manipulation/négociation - opère sur les parties censées constituer ensuite une totalité, voire une réponse convaincante et stable aux questionnements. Certes, ici la totalité n'est qu'en voie de constitution - la totalité de l'objet de recherche, on le rappelle, est identifiable comme la chaîne complète des images ${ }^{11}$, qui garantit la solidité de la référence.

Dans une autre chaîne d'images contenue dans l'article examiné (Figure 3), nous sommes en présence d'une prolifération d'images produites par d'autres images. Contrairement à la série précédente, ces images n'ouvrent pas sur des nouvelles scènes à travers un fonctionnement « à fenêtre » mais effectuent des sélections et des tris.

A

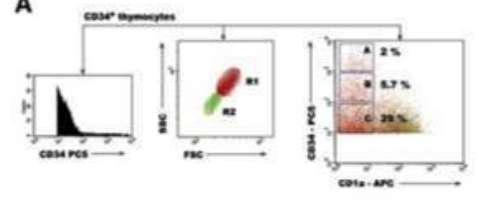

B

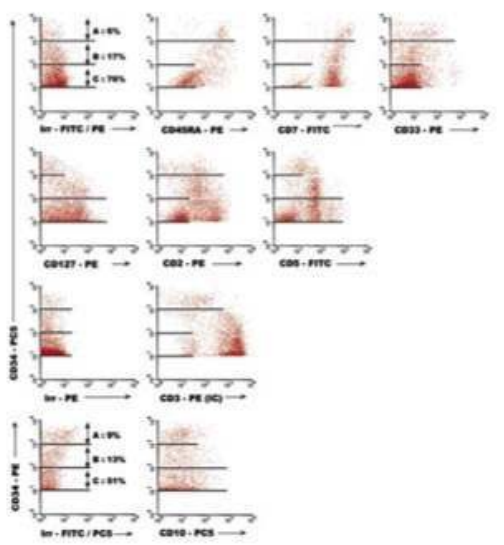

c

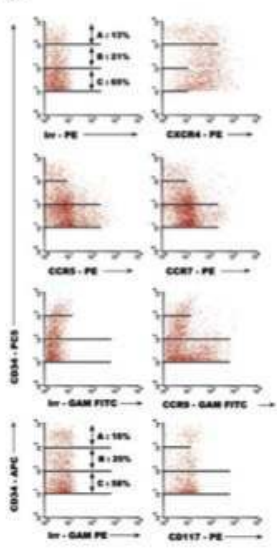

Figure 3. «Phenotypic and Molecular Characterization of Immature CD34+CD1a2/+ Fetal Thymocytes » (Haddad et alii 2006 : 223), detail.

Dans la série $\mathrm{A}$, la toute première image à gauche, qui fait partie d'une série de trois images, met en scène une population de cellules sélectionnées (cellules étant susceptibles de coloniser le thymus), tandis que la deuxième image montre les différentes tailles des cellules de cette population. La troisième image trie encore, car elle ne rend compte que du développement temporel des cellules d'une seule taille, représentées en rouge. Cette méthode d'enchaînement d'images ne concerne pas des opérations de focalisation ou de superposition, mais bien de division et de tri.

D'autres images publiées dans l'article étudié nous présentent des nouvelles manipulations de l'objet de recherche (figure 4). 

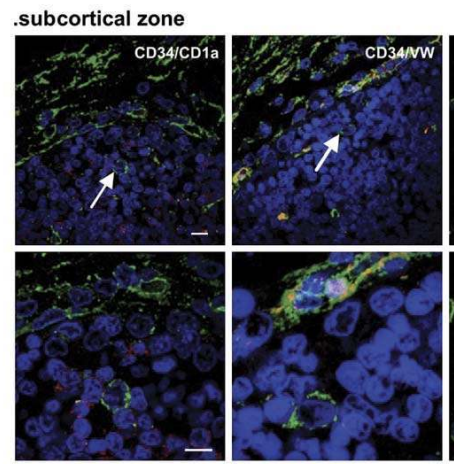

cortico-medular junction
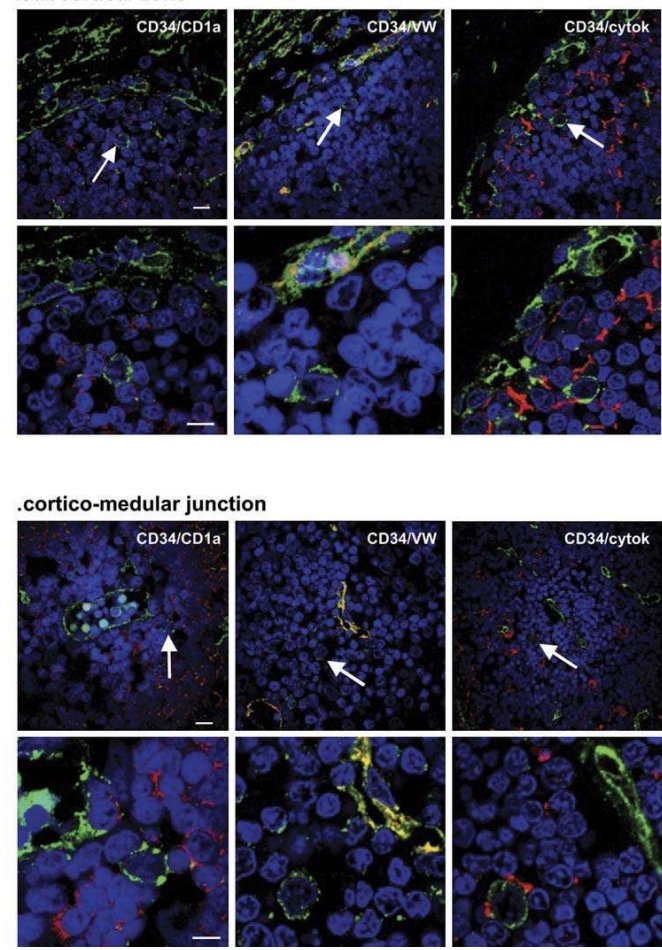

C
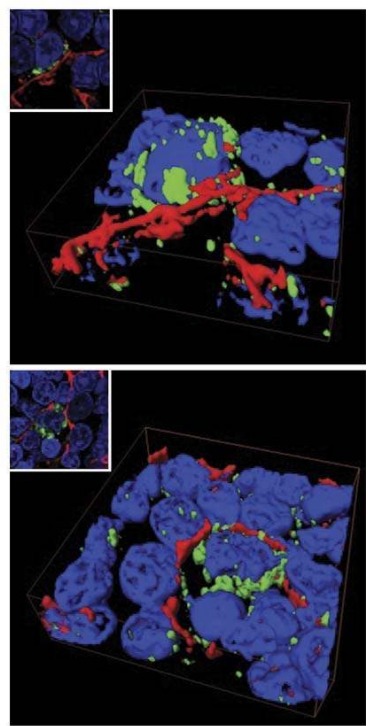

Figure 4. «Subanatomic Localization of Early Thymus Immigrants » (Haddad et alii, 2006 : 224).

Examinons tout d'abord les groupes A et B. Dans les deux séries, les images du groupe A constituent une micro-totalité par rapport aux images du groupe B qui sont des agrandissements des cellules souches repérées au sein des vaisseaux constituant le tissu du thymus.

Dans les images du groupe A, les cellules souches sont identifiées par la flèche et colorées en vert. Il faudrait se demander pourquoi, dans le but de focaliser l'attention sur ces cellules, les chercheurs n'ont pas utilisé des cadrages rectangulaires ou des carrés mais bien une flèche. C'est en raison du fait que, contrairement à la première image qu'on a décrite (Figure 2, groupe A), dans la figure 4 une seule cellule apparaissant dans le tissus du thymus est visée et non pas un paysage de cellules. Dans la première série d'images (Figure 2, groupe A), on était face à une ouverture rectangulaire car un paysage était visé, voire des dispositions de cellules et des patterns ; ici, en revanche, c'est une cellule individuelle qui fait l'objet de l'exploration.

Si, dans le premier cas examiné (Figure 2), ce sont des patterns qui sont visés et le cadre du carré produisant une image-paysage s'avère nécessaire, ici en revanche, c'est une individualité qui est visée, voire quelque chose qu'on peut pointer du doigt et qui relève donc plutôt d'une composition que nous appellerions volontiers image-portrait. D'un côté, on a une image-paysage visant la représentation de la distribution d'une multiplicité de cellules par un cadrage, de l'autre on a une image-portrait visant la mise en valeur d'une « unicité » et non plus la « distribution d'une multiplicité ».

Ce qui se passe dans la série $C$ est aussi digne d'intérêt (Figure 5). 


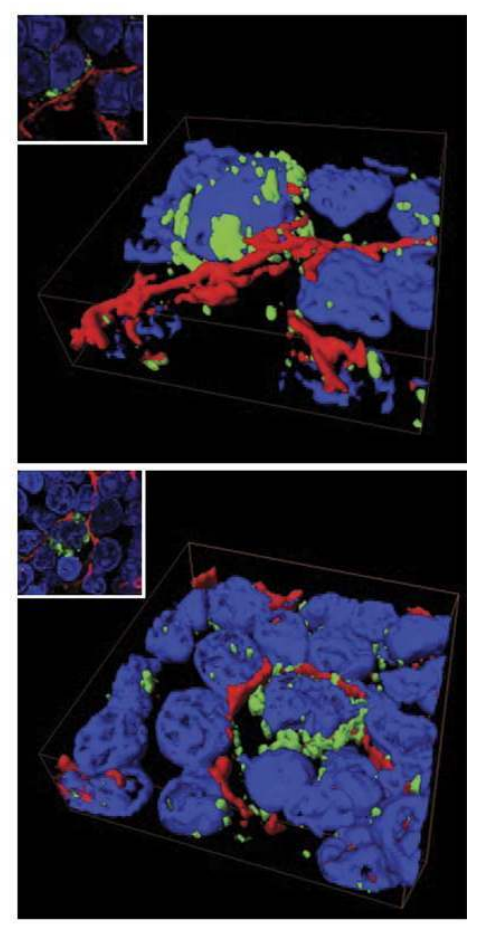

Figure 5. Détail de la figure 4 (groupe C). 


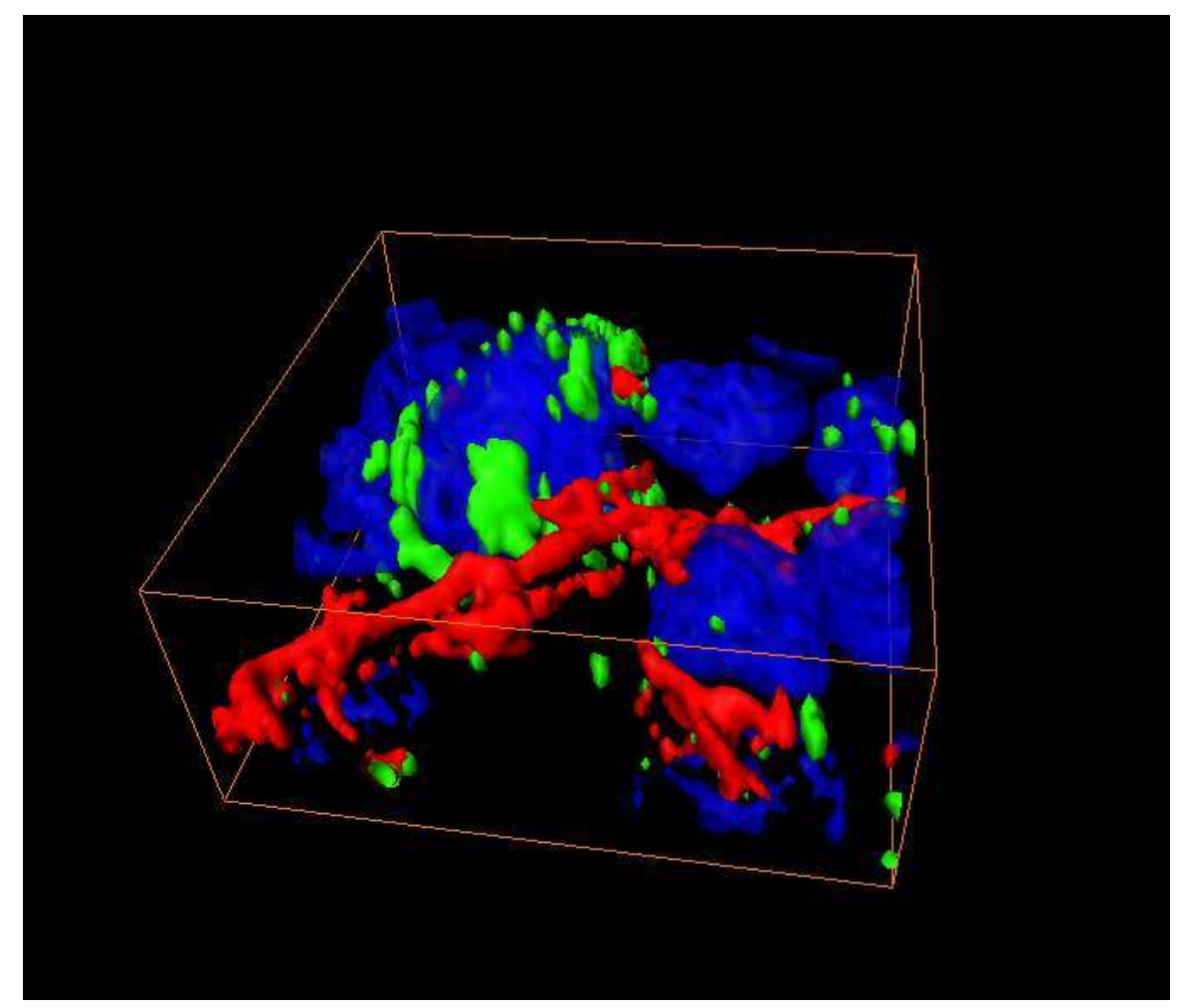

Figure 6. Complément de la figure 5. Image non publiée dans l'article en question'2.

49 Il s'agit ici d'un tout autre genre de cadrage: il n'a pas exclusivement une fonction isolante ou focalisante, mais il fonctionne également comme contenant, comme acte d'englobement, voire comme une enveloppe. Si l'image bidimensionnelle fonctionne comme une surface, presque à l'instar d'une carte, l'image tridimensionnelle vise à donner corps à la cellule. On se déplace ainsi de l'abstraction de la carte de l'image bidimensionnelle vers la représentation de la corporéité de la cellule. Comme elle est en train de se développer, voire de se différencier, elle est rendue de manière tridimensionnelle à l'instar d'un corps en transformation.

\section{Les opérations rhétoriques}

À la suite de ce travail d'analyse, nous pouvons identifier cinq opérations méréologiques, bien que, comme nous l'avons dit dans les prémisses épistémologiques ainsi que dans l'analyse de la figure 2, c'est dans la chaîne de transformations des images, dans l'intervalle entre une image et l'autre, que les opérations les plus intéressantes de projection/remémoration sont à l'œuvre.

51 Les cinq opérations méréologiques que nous pouvons isoler de notre corpus sont donc les suivantes :

- 1. Focaliser/ Centrer/Distribuer les centres de l'attention

- 2. Projeter/Explorer/Devancer

- 3. Superposer/Envahir l'espace de l'observateur pour construire une évidence

- 4. Trier/Diviser

- 5. Croiser les visions/ additionner/vérifier 
On s'aperçoit que les images interviennent dans le processus de l'expérience scientifique en tant qu'opérations régulatrices ordonnant un certain nombre de phénomènes. Dans le flux de l'expérimentation, des totalités se constituent, mais de manière provisoire car elles continuent à se faire et se défaire en parties, identifiables bien que changeantes. Ce processus qui vise la stabilisation de formes en une totalité acceptée par la communauté scientifique peut recevoir une description syntagmatique de ce genre :

< Flux => Inflexion => Parties saillantes => Identification et différentiation => Conjonction et disjonction $\Rightarrow>$ Composition et décomposition de parties $=>$ Emergence et disparition des genres (ressemblances de famille, patterns) => Formation et déformation de séries $>^{13}$

Les chaînes d'images expérimentales que nous avons étudiées nous offrent chacune des "réponses partielles" qui se caractérisent comme des approximations et des tâtonnements successifs dont il faut tester la commensurabilité pour les présenter comme valables et identifiant une totalité d'objet. D'ailleurs, c'est précisément la problématique de l'objet scientifique en tant que totalité à construirequi justifie dans notre analyse la référence à la rhétorique visuelle et à la méréologie. D'une certaine façon, on pourrait affirmer que le parcours allant de l'objet de recherche comme interrogation jusqu'à l'objet scientifique comme totalité acceptée - au moins par une partie de la communauté scientifique - se déploie à partir d'un état de questionnement en se dirigeant vers un état de partage communautaire et institutionnalisé ${ }^{14}$.

\section{Le cas de la vulgarisation}

Dans le processus de stabilisation de formes que nous avons suivi jusqu'à présent, les images qui testent l'objet de recherche sont à entendre comme différentes compositions de l'objet de recherche résultant de l'expérimentation. C'est pour cela que l'objet de recherche n'est jamais figé dans une seule image mais bien dans une chaîne qui demande à être testée/vérifiée. Certes, chaque article peut choisir la densité d'enchaînement à valoriser. Souvent, en effet, des collègues concurrents peuvent objecter sur cette densité et sur le possible manque d'une étape. La chaîne complète des images produites en laboratoire ne peut d'ailleurs jamais être présente en entier dans un article. Cependant, si l'article de recherche réduit massivement les images utilisées dans les laboratoires pour le présenter au public, dans le cas de la vulgarisation, la réduction est tellement drastique que le résultat est souvent celui de la réification d'un objet ou d'un processus en une seule image.

À ce propos, nous examinons un cas de vulgarisation et notamment un manuel qui a pour titre Immunobiologie, écrit par Charles Janeway et alii (1997).

Les images publiées dans le chapitre consacré aux cellules du thymus (Figure 7) sont légendées d'un titre assez long qui fonctionne en effet comme un résumé de l'explication qui se déploie dans le texte à côté: "Les cellules $\mathrm{T}$ prennent aussi naissance dans la moelle osseuse, mais tous les événements importants de leur développement surviennent dans le thymus ». 


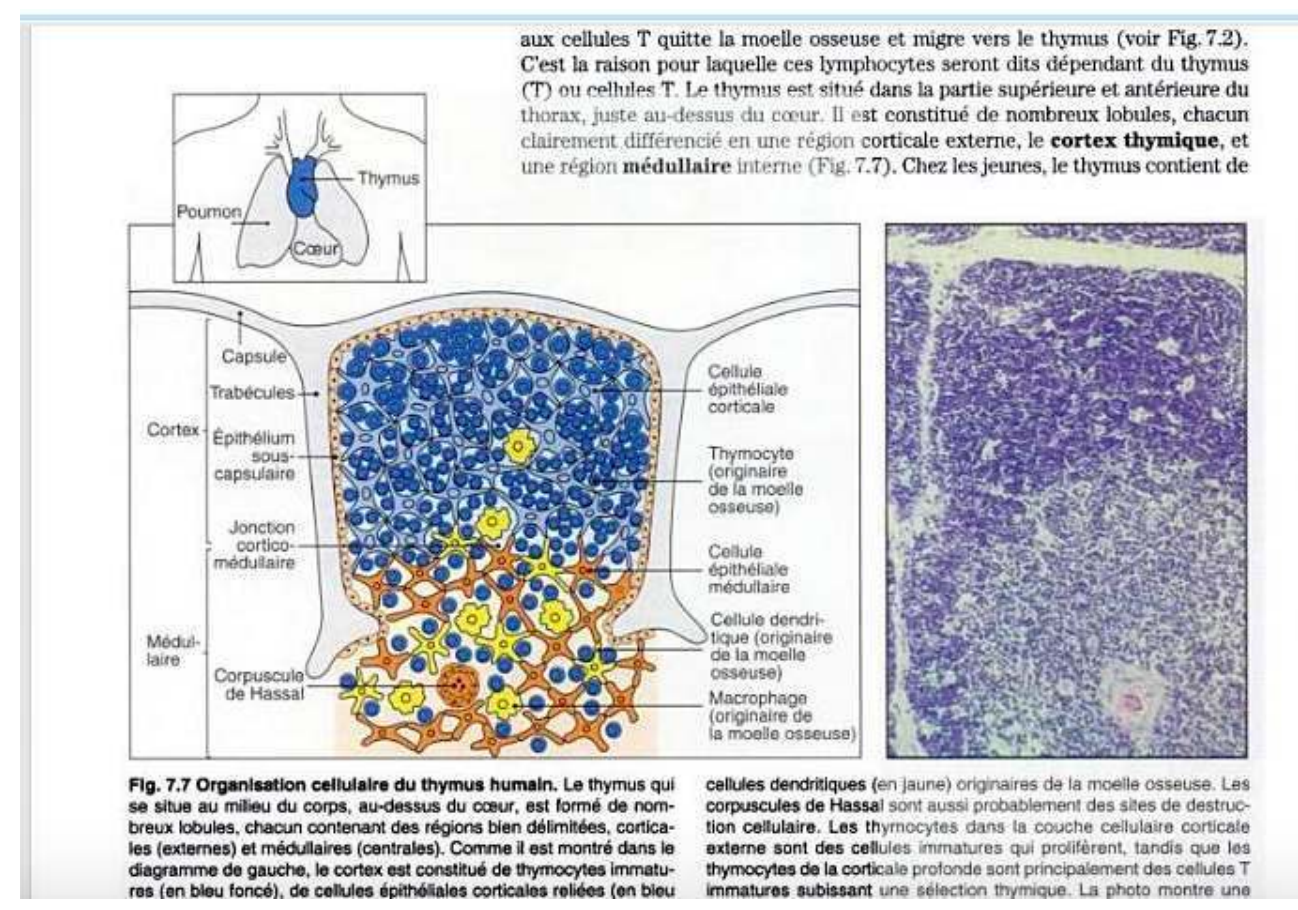

Figure 7. « Les cellules T prennent aussi naissance dans la moelle osseuse, mais tous les événements importants de leur développement surviennent dans le thymus » (Janeway et alii, 1997 : 231).

Dans ces pages de manuel, on aborde la même question que dans l'article de recherche, à savoir la naissance de certaines cellules dans la moelle osseuse se déplaçant dans le thymus où elles se différencient; dans ce genre discursif, pourtant, le cadrage de la matière pertinente est beaucoup plus large que dans l'article de recherche.

Nous nous apercevons que le manuel « fait un pas en arrière » par rapport à l'article de recherche : dans la figure 7, tout en haut, un petit schéma localise le thymus dans le corps humain, tandis que plus bas, nous observons deux images disposées l'une à côté de l'autre. La première (de gauche à droite) montre schématiquement l'organisation cellulaire du thymus; elle est accompagnée d'une image photographique qui, étant une empreinte, s'en manifeste comme la source. La disposition des deux images l'une à côté de l'autre vise à surligner qu'elles (l'image microscopique et le schéma explicatif) sont des traductions réciproques. Contrairement à l'article de recherche, on n'est plus face à une chaîne de médiations visuelles qui vont vers l'avant, d'une transformation à l'autre, mais on est confrontés à un rapport dialogique entre deux images visant à montrer des équivalences. En effet, deux syntaxes figuratives se juxtaposent. L'association de l'image et du schéma offre une vision interstitielle qui permet d'apercevoir une possible transposabilité de relations d'un système de représentation à l'autre.

La chaîne d'images qui caractérisait le parcours de l'exploration dans l'article de recherche s'est ainsi arrêtée. La référence apparaît comme stabilisée car les images sont disposées de manière à montrer des équivalences à l'intérieur d'un système de comparaison clôturée: aucun mouvement de relance n'est présent en vue d'une poursuite de l'exploration. Il s'agit plutôt d'une transposition visant à trouver des correspondances/équivalences entre un système de représentation et l'autre, annulant tout renvoi au processus expérimental.

60 Le schéma se réfère à l'image microscopique qui le justifie et l'image microscopique est expliquée par le schéma. Une image sert de référence à l'autre. L'image microscopique 
a été isolée de la chaîne de transformations qui l'a conçue, elle a subi un processus de "séparation ». Elle est réduite à un produit immuable, et n'est plus susceptible d'être (re)déployée par d'autres chercheurs car les paramètres de contrôle permettant les manipulations expérimentales ne sont pas affichés : le couple d'images est présenté ici comme "définitif ». L'acte de fabrication reste caché : c'est pourquoi le thymus résulte comme réifié dans une image microscopique qui trouve son pendant dans un schéma. Le texte verbal qui entre dans le schéma montre également le fait que l'organisation des cellules dans le thymus est considérée comme stabilisée.

61 Nous tenons à remarquer ici que la disposition du texte dans l'article scientifique et dans le manuel relève de deux types d'explication différents. Les textes verbaux de l'article de recherche s'enchaînent de la même manière que les images, en continu, tandis que, dans le manuel, la disposition des images en dialogue figeant la référence fait en sorte que le texte verbal puisse entrer dans le cadre de l'image afin de localiser/ réifier les parties qui composent le thymus. Les images de la vulgarisation, en excluant le rappel des échelles et des valeurs mathématiques, en effaçant les procédés de la fabrication et en accueillant le texte verbal, offrent finalement au lecteur un objet de recherche réifié, achevé.

Les images qui suivent mettent en scène un autre procédé de la vulgarisation, en mettant en scène les souris, voire les cobayes des expériences en laboratoire (Figure 8).

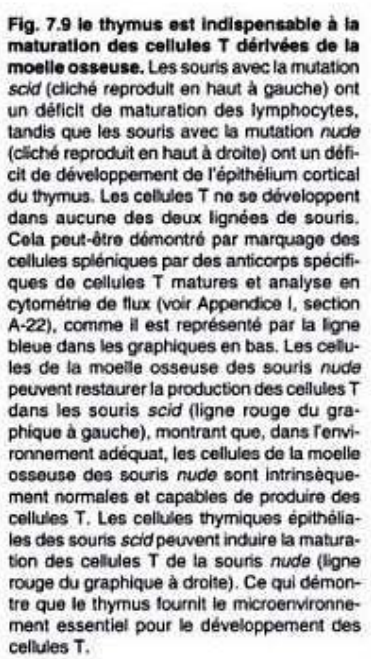
cellules $\mathrm{T}$.
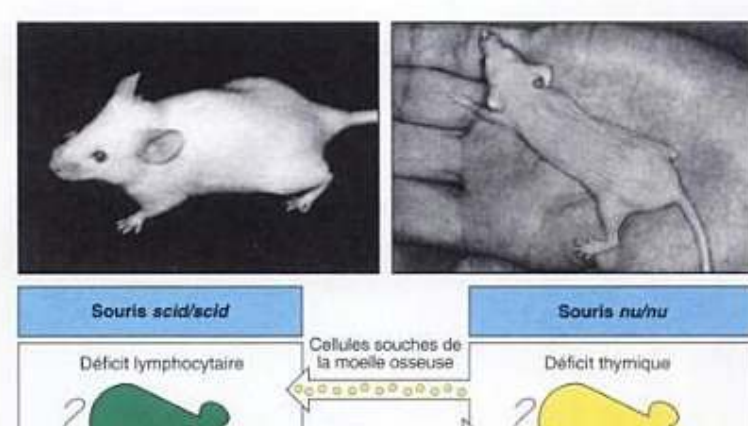

Souris numu Dofict thymique Deficit thymique Deficit thym
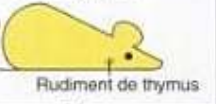

Grette de thymus
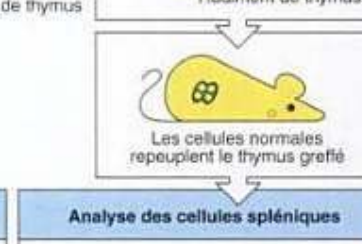

Analyse des cellules spléniques

de cellules Nambre
de cellules

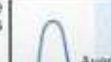

Figure 8. «Le thymus est indispensable à la maturation des cellules T dérivées de la moelle osseuse » (Janeway et alii, $1997: 23$ ).

Les souris sont à la base des expériences de l'article de recherches (en effet, les cellules sont extraites du sang placentaire humain et ensuite greffées sur la souris) mais les souris ne sont montrées que dans les manuels de vulgarisation destinés aux étudiants. Si les images de la vulgarisation ne visualisent pas les opérations mais tout simplement des équivalencesqui bloquent toute possibilité de mise en chaîne, en revanche les souris sont mises en scène en tant que support des expériences. Les opérations de laboratoire 
sont donc remplacées par la présentation des éléments composant les expériences passées - ce dont les expériences sont faites.

\section{Conclusion}

64 La vulgarisation couvre une extension de la matière bien plus large que les genres discursifs de la recherche et, d'une certaine manière, elle permet aussi la prise de distance et une hauteur de survol car elle met en scène les instruments de l'expérimentation, absents des articles scientifiques.

Nous pourrions enfin affirmer que les textes de la vulgarisation mettent en scène toutes les composantes de la recherche, tous les instruments qui y sont nécessaires; autrement dit, ils les présentent au public étudiant qui doit en apprendre la grammaire d'utilisation. En revanche, aucune syntagmatique du processus scientifique n'est décrite; nous sommes simplement face à une paradigmatique de l'usage et plus précisément à l'identification des outils, voire des instruments qui seront nécessaires afin d'entamer les expériences à venir. Dans l'article de recherche, en revanche, les outils sont connus du public d'experts et il serait inutile de les représenter; c'est le déploiement des outils qui est mis en scène et notamment les étapes du processus d'exploration - qui doit être décrit avec le plus de densité possible pour que les processus puisse apparaitre comme une totalité difficile à désagréger.

\section{BIBLIOGRAPHY}

Allamel-Raffin, Catherine. 2005. « De l'intersubjectivité à l'interinstrumentalité. L'exemple de la physique des surfaces ", Philosophia Scientiae. Travaux d'histoire des Sciences et de Philosophie 9, 3-30 Bastide, Françoise. 1979. «Le foie lavé, approche sémiotique d'un texte de sciences expérimentales » (Paris : Groupe de recherches sémio-linguistiques, EHESS, CNRS)

Bastide, Françoise. 1985. « Iconographie des textes scientifiques. Principes d'analyse », Culture 14, 132-151

Bastide, Françoise. 2001. Una notte con Saturno. Scritti semiotici sul discorso scientifico (Rome : Meltemi)

Bordron, Jean-François. 2009. « Expérience d'objet, expérience d'image », Visible 5, 111-122

Bordron, Jean-François. 2010. « Rhétorique et économie des images », Protée 38, 27-40

Bordron, Jean-François. 2011. L'iconicité et ses images (Paris : PUF)

Bordron, Jean-François. 2012. « Image, événement, présupposition », Visible 8, 105-121

Bordron, Jean-François. 2013. Image et vérité. Essais sur les dimensions iconiques de la connaissance (Liège : PULg)

Dondero, Maria Giulia. 2009. «The Semiotics of Scientific Image : from production to manipulation », The American Journal of Semiotics 25, 1-19 
Dondero, Maria Giulia. 2010. «Sémiotique de l'image scientifique », Signata- Annales des sémiotiques/Annals of Semiotics 1, 111-176

Dondero, Maria Giulia. 2016. «L'énonciation énoncée dans l'image », Marion Colas-Blaise, Laurent Perrin \& Gian Maria Tore (éds), L'énonciation aujourd'hui (Limoges : Lambert Lucas), 343-369.

Dondero, Maria Giulia \& Fontanille, Jacques. 2012. Des images à problèmes. Le sens du visuel à l'épreuve de l'image scientifique (Limoges : Pulim)

Fontanille, Jacques. 1989. Les Espaces subjectifs. Introduction à la sémiotique de l'observateur (Paris : Hachette)

Groupe $\mu .1992$. Traité du signe visuel. Pour une rhétorique de l'image (Paris : Seuil)

Haddad, Rima et alii. 2006. « Dynamics of Thymus-Colonizing Cells during Human Development », Immunity 24, 217-230

James, William. 2007. Essais d'empirisme radical (Paris : Flammarion)

Janeway, Charles A., Paul Travers, Mark Walport \& Mark J. Shlomchik. 1997. Immunobiologie

(Paris : De Boeck)

Latour, Bruno. 1999. Pandora's Hope: An Essay on the Reality of Science Studies (Cambridge: Harvard University Press); tr. fr. L'espoir de Pandore. Pour une version réaliste de l'activité scientifique, 2001 (Paris : La Découverte)

Latour, Bruno. 2012. Enquête sur les modes d'existence (Paris : La Découverte)

Latour, Bruno \& Emilie Hermant. 1998. Paris Ville Invisible (Paris : Synthelabo)

Stoichita, Victor. 1993. L'instauration du tableau. Métapeinture à l'aube des temps modernes (Paris : Méridiens Klinksieck)

\section{NOTES}

1. Organe lymphoïde situé derrière le sternum, devant la trachée, et dont le volume diminue après la deuxième année de la vie ; il joue un rôle très important dans la mise en place du système immunitaire.

2. Nous avons réalisé un séjour de recherche à l'Institut universitaire d'Hématologie (Paris 7) en 2014 sous la direction de Bruno Canque. Nous tenons à remercier Bruno Canque et Kutaiba Alhaj Hussen pour les explications et les échanges au sujet de ce travail.

3. Voir plus loin les figures 2 à 7 .

4. Pour des raisons d'espace, nous ne nous attarderons pas ici sur la sémiotique mathématique de Charles Sanders Peirce concernant les diagrammes en tant que visualisations textualisées et/ ou mentales, ni sur la philosophie des mathématiques de Gilles Châtelet concernant le diagramme comme dispositif porteur de nouvelles connaissances. Nous n'aborderons pas non plus la théorie des systèmes symboliques autographiques et allographiques formulée par Nelson Goodman qui a pourtant été à la base de nos réflexions sur le discours scientifique. Pour un panorama complet sur ces théories, voir Dondero et Fontanille (2012).

5. Françoise Bastide, biologiste, a été formée à la sémiotique de Greimas. Elle nous a laissé un certain nombre d'articles importants, publiés dans les années 1970 et 1980 dans des revues telles que Actes Sémiotiques, Protée, Sociétés, Culture Technique, Cahiers Science-Technologie-Société. Ces articles n'ont malheureusement été réunis qu'en Italie, traduits en italien, sous la direction de B. Latour et avec une préface de P. Fabbri (Bastide 2001). 
6. Chaque image contient des simulacres de l'acte de production et d'observation (énonciation énoncée). À ce sujet, voir Fontanille (1989), Dondero (2016).

7. Sur l'inter-instrumentalité, voir Allamel-Raffin (2005).

8. La théorie de Jean-François Bordron concernant l'iconicité en tant que méréologie est illustrée par ses deux derniers livres $(2011 ; 2013)$.

9. Haddad et alii, Immunity 24, pp. 217-230, février 2006.

10. Voir à ce propos Stoichita (1993).

11. Sur la solidité de la référence en tant que totalité qu'il est possible de parcourir dans les deux sens (le sens du processus qui l'a généré et le sens inverse, celui de la vérification de cet enchaînement de la part des collègues), voir Latour (1999, et notamment «Sol amazonien et circulation de la référence »).

12. Nous remercions B. Canque de nous avoir fourni cette image qui nous permet de voir la grille tridimentionelle qui est moins définie dans la figure 4.

13. J'emprunte cette schématisation à Bordron (2012) qui s'inspire de James (2007).

14. Techniquement parlant, le parcours envisagé va de ce qu'on appelle indicialité à ce qu'on appelle symbolisation en passant par l'iconisation. Il s'agit de termes empruntés à la théorie de C.S. Peirce, revisitée par Bordron (2011). Dans le premier moment, celui de l'indicialité entendue comme interrogation, l'image est encore virtuelle mais on prépare son lieu d'apparition, voire un référentiel grâce auquel ce qu'on cherche peut trouver des conditions de manifestation; dans le second moment, celui de l'iconisation, les images cherchent à se stabiliser en tant que réponses à l'interrogation, et dans le troisième moment, celui de la symbolisation, qui est le lieu de la stabilisation et de la codification, nous allons vers le régime de la vulgarisation. Voir à ce propos Dondero $(2009 ; 2010)$.

\section{ABSTRACTS}

This article examines the rhetoric of images in the biological sciences. The epistemological context of our study is found in Bruno Latour's sociology of science and Françoise Bastide's semiotics of scientific images, in the theory and methodology of post-Greimassian discourse semiotics, and in the rhetorical theories of Groupe $\mu$ and Jean-François Bordron. We use these different perspectives in an analysis of rhetorical operations (addition, deletion, selection, superposition, and more) performed by laboratory scientists, as represented in our corpus of images. Our particular focus is on a rhetoric that is "mereological", so named because it is based on the relation between the whole and the parts.

Cet article porte sur la rhétorique des images en sciences biologiques. Le cadre épistémologique de notre travail provient de la sociologie des sciences de Bruno Latour et de la sémiotique de l'image scientifique de Françoise Bastide, de l'orientation théorique et méthodologique de la sémiotique du discours post-greimassienne, ainsi que des théories rhétoriques du Groupe $\mu$ et de Jean-François Bordron. À travers ces différentes approches, nous visons à analyser les opérations rhétoriques accomplies par les scientifiques en laboratoire (addition, suppression, sélection, superposition, etc.) dont témoigne notre corpus d'images. Il s'agit notamment d'une rhétorique que nous appellerons « méréologique » car fondée sur la relation entre totalité et parties. 
INDEX

Keywords: enunciation, mereology, rhetoric, scientific discourse, semiotics of images

Mots-clés: discours scientifique, énonciation, méréologie, rhétorique, sémiotique de l'image

\section{AUTHOR}

\section{MARIA GIULIA DONDERO}

Fonds National de la Recherche Scientifique ; Université de Liège 